\title{
Consulta en servicio de urgencia y reingreso hospitalario después de una cirugía bariátrica primaria
}

\author{
Rodrigo Muñoz C. ${ }^{1}$, Paula León A. ${ }^{1}$, Rodrigo Tejos S. ${ }^{1}$, Nicolás Quezada ${ }^{1}$, Pablo Achurra T. ${ }^{1}$, \\ Mauricio Gabrielli ${ }^{1}$, Gustavo Pérez ${ }^{1}$, Luis Ibáñez ${ }^{1}$ y Fernando Crovari E. ${ }^{1}$
}

\section{Emergency department visit and Hospital Readmission after Primary Bariatric Surgery}

Introduction: Emergency department visits (EDV) and hospital readmission (HR) after bariatric surgery (BS) are important indicators of quality and safety in surgery, however there is little information on their characteristics in our national environment. Aim: In this work we analyze EDV and HR in patients undergoing a primary BS in a high-volume university center, and identify variables that could be associated with these indicators. Materials and Method: A retrospective observational study where we identified all patients undergoing Roux-in-Y gastric bypass (RYGBP) or primary laparoscopic sleeve gastrectomy (SG) performed consecutively at our institution during the period 2006-2007 and 2012-2013. Using our clinical records and prospective follow-up database, we identify those patients with EDV and/or HR in our hospital during the first 30 days after discharge. Results: 1146 primary BS were included, of these $53 \%(\mathrm{n}=613)$ were RYGBP and $47 \%(n=533)$ SG. 8,03\% $(n=92)$ of the patients had at least one EDV, of these 3,7\% $(n=42)$ had an HR. The independent variables associated with EDV and HR were the operative time and preoperative body mass index (BMI). No statistical association was found, in the period studied, for the type of BS performed with EDV or HR. Conclusion: There is a low proportion of patients who require EDV and HR after BS, which demonstrates the safety of these interventions.

Keywords: Roux Y gastric bypass; sleeve gastrectomy; readmission; emergency department visit.

\section{Resumen}

Introducción: Las consultas en el servicio de urgencia $(\mathrm{CU})$ y el reingreso $(\mathrm{RI})$ hospitalario después de una cirugía bariátrica (CB) son importantes indicadores de calidad y seguridad. Sin embargo, existe escasa información de estos indicadores en nuestro medio nacional. En este trabajo analizamos las CU y RI de pacientes sometidos a una CB primaria en un centro universitario de alto volumen, y buscamos variables asociadas a estos indicadores. Materiales y Método: Estudio observacional retrospectivo que incluyó a todos los pacientes sometidos a bypass gástrico (BPG) o gastrectomía en manga (GM) laparoscópica primaria realizados de forma consecutiva en nuestra institución durante el período 2006-2007 y 20122013. Utilizando nuestros registros clínicos y base de seguimiento prospectivo, identificamos aquellos pacientes con $\mathrm{CU}$ o RI en nuestro hospital durante los primeros 30 días después del alta. Resultados: Se incluyeron $1.146 \mathrm{CB}$ primarias, 53\% $(\mathrm{n}=613)$ fueron BPG y 47\% $(\mathrm{n}=533)$ GM. Un 8,03\% $(\mathrm{n}=92)$ de los pacientes tuvo al menos una CU y un 3,7\% $(n=42)$ un RI. Las variables independientes asociadas tanto a CU como RI fueron el tiempo operatorio e índice de masa corporal (IMC) preoperatorio. No se encontró asociación estadística, en el periodo estudiado, para el tipo de CB realizada con la CU ni con el RI. Conclusión: Existe una baja proporción de pacientes que requieren CU y RI posterior a la $\mathrm{CB}$, lo que demuestra la seguridad de estas intervenciones.

Palabras clave: bypass gástrico en Y de Roux; gastrectomía en manga; readmisión; consulta urgencia.
'Departamento de Cirugía Digestiva, Escuela de Medicina, Pontificia Universidad Católica. Santiago, Chile.

Recibido 2020-10-30, aceptado 2020-02-08

Correspondencia a: Dr. Rodrigo Muñoz C. rmunozc@med.puc.cl 


\section{Introducción}

La cirugía bariátrica $(\mathrm{CB})$ tiene excelentes resultados logrando una baja de peso significativa y sostenida en el tiempo, además de una mejoría o resolución de múltiples comorbilidades. Adicionalmente, puede incluso prevenir la aparición de otras enfermedades. En conjunto, estos beneficios aumentan la expectativa de vida en pacientes sometidos a estas cirugías ${ }^{1}$. En la actualidad, la CB tiene una baja morbimortalidad comparable a la de otros procedimientos quirúrgicos menos complejos ${ }^{2,3}$. De igual manera, las tasas de consulta en urgencia (CU) y de reingreso (RI) hospitalario son bajas después de este tipo de intervenciones ${ }^{4-7}$.

Actualmente, tanto la CU como el RI son importantes indicadores de calidad y seguridad en cirugía. Ambos, pueden aumentar de manera inesperada los costos de una $\mathrm{CB}$, por lo que es importante identificar variables asociadas a ambos indicadores ${ }^{8}$. Hoy se estima que las tasas de CU y RI durante los primeros 30 días después de una $\mathrm{CB}$ pueden variar entre un 5\%-10\% ${ }^{9-11}$, dependiendo del tipo de CB. Así, cirugías como el bypass gástrico (BPG) tienden a tener mayores tasas de CU y RI que las observadas después de una gastrectomía en manga (GM). Afortunadamente, una proporción menor de los pacientes que acuden al servicio de urgencias (SU) requiere finalmente una hospitalización ${ }^{12}$, lo que sugiere que algunas CU podrían haber sido prevenidas ${ }^{13,14}$.

El objetivo principal de nuestro trabajo fue analizar las CU y los RI hospitalarios durante los primeros 30 días después de una $\mathrm{CB}$ primaria en un centro universitario de alto volumen. Adicionalmente, buscamos factores que pudieran estar asociadas a estos indicadores.

\section{Materiales y Método}

\section{Diseño de estudio}

Estudio observacional retrospectivo. Se identificaron a todos los pacientes que fueron sometidos a BPG o GM primaria por vía laparoscópica, de forma consecutiva, en el Hospital Clínico de la Universidad Católica de Chile durante 2 periodos de 2 años cada uno (2006-2007 y 2012-2013). Se incluyeron en este estudio a todos los pacientes que tuvieron al menos una $\mathrm{CU}$ y/o RI en nuestra red de salud durante los primeros 30 días posteriores al alta. La información fue obtenida a partir de nuestros registros médicos impresos y electrónicos, así como también a partir de nuestra base de datos de seguimiento prospectiva. Las complicaciones quirúrgicas Clavien-Dindo $\geq 3$ fueron registradas. Este estudio fue aprobado por el comité de ética de nuestra institución.

\section{Definición de consulta en servicio de urgencia y reingreso hospitalario}

Definimos CU como una presentación en el SU por cualquier motivo durante los primeros 30 días después del alta. El RI se definió como cualquier visita al SU que concluyó con la hospitalización del paciente independiente de si fuera necesario o no la realización de algún tipo de intervención.

\section{Técnica quirúrgica}

\section{$B P G$ laparoscópico}

Nuestra técnica quirúrgica fue descrita con anterioridad $^{15}$. En resumen, el yeyuno es seccionado entre 20 a $30 \mathrm{~cm}$ distal al ángulo de Treitz, con mínima sección mesentérica, creando un asa alimentaria de $150 \mathrm{~cm}$ para posteriormente realizar yeyuno-yeyuno anastomosis mecánica. Se confecciona una bolsa gástrica usando una sonda de calibración de $34 \mathrm{Fr}$ de aproximadamente $5 \mathrm{~cm}$ de longitud. A continuación, se realiza una gastro-yeyuno anastomosis manual en dos planos. Las brechas mesentéricas y el espacio de Petersen son cerrados de forma rutinaria.

\section{Gastrectomía en manga}

La GM se realiza por medio de una resección tubular del estómago con un dispositivo de grapado mecánico de acuerdo con nuestra técnica previamente reportada ${ }^{16}$. La calibración se realiza con un tubo de silicona de 34-50 Fr, según la preferencia del cirujano, con el objetivo de conseguir un reservorio gástrico de aproximadamente $200 \mathrm{ml}$. Una vez resecado el estómago, la línea de grapas se refuerza de forma manual con una sutura sintética absorbible.

\section{Tromboprofilaxis}

En nuestra institución, el protocolo de tromboprofilaxis consiste en: uso de medias antitrombóticas instaladas al ingreso del paciente a pabellón, uso de medias de compresión neumática intermitente durante la cirugía, las que se mantienen durante las primeras $24 \mathrm{~h}$, y quimioprofilaxis que se inicia durante las primeras 6-8 h después de terminada la cirugía con heparina de bajo peso molecular. En base a los factores de riesgo del paciente, la tromboprofilaxis se indica para el posoperatorio de acuerdo con las recomendaciones actuales ${ }^{17}$.

\section{Análisis estadístico}

Las variables continuas se presentan como promedio y desviación estándar, las que fueron 
Tabla 1. Características generales del grupo estudiado

\begin{tabular}{|lcccc|}
\hline Características & Total $\mathbf{n}=\mathbf{1 . 1 4 6}$ & $\mathbf{G M}=\mathbf{5 3 3}$ & BPG $=\mathbf{6 1 3}$ & Valor p \\
Sexo, $\mathrm{n}(\%)$ & & & & \\
$\quad$ Mujeres & $836(73 \%)$ & $404(76 \%)$ & $433(71 \%)$ & NS \\
$\quad$ Hombres & $310(27 \%)$ & $129(24 \%)$ & $180(29 \%)$ & \\
Edad (años)* & $36,8 \pm 11,7$ & $36,1 \pm 11,6$ & $37,6 \pm 11,7$ & NS \\
IMC preoperatorio $\left(\mathrm{kg} / \mathrm{m}^{2}\right)^{*}$ & $37,5 \pm 5,5$ & $35,5 \pm 4,4$ & $39,3 \pm 5,6$ & 0,01 \\
DM2 preoperatorio, n (\%) & $275(24 \%)$ & $80(15 \%)$ & $195(32 \%)$ & 0,01 \\
Hipertensión preoperatorio, n (\%) & $190(16 \%)$ & $41(8 \%)$ & $90(15 \%)$ & 0,01 \\
Dislipidemia preoperatorio, n (\%) & $131(11 \%)$ & $60(11 \%)$ & $130(21 \%)$ & 0,01 \\
Tiempo operatorio* (min) & $97 \pm 37$ & $77 \pm 26$ & $115 \pm 36$ & 0,01 \\
Días hospitalización* $^{*}$ & $3,3 \pm 5,5$ & $2,9 \pm 1$ & $3,6 \pm 7,5$ & 0,03 \\
\hline
\end{tabular}

*Promedio/desviación estándar. N.S: no significativo.

comparadas usando la prueba de T de Student. Las variables categóricas se presentan como porcentajes y fueron comparadas usando la prueba de chi-cuadrado y test exacto de Fisher. El análisis univariado se realizó para identificar los factores asociados a CU y RI. Los factores identificados a través del análisis univariado se usaron en un modelo de regresión logística multivariada. Un valor de $\mathrm{p}<0,05$ se consideró significativo. El análisis estadístico se realizó utilizando software disponible comercialmente (SPSS 15 para Windows, Inc., Chicago, IL, EE. UU).

\section{Resultados}

En total se incluyeron 1.146 pacientes, 613 (53\%) BPG y 533 (47\%) GM en este estudio. La edad e IMC preoperatorio promedio fue de $36,8 \pm 11,7$ años y $37,5 \pm 5,5 \mathrm{~kg} / \mathrm{m}^{2}$, respectivamente. En relación a la presencia de comorbilidades, se presentaron diferencias estadísticamente significativas entre los 2 tipos de cirugías que se relacionan con las indicaciones de cada una de ellas. La Tabla 1 resume las características del total de pacientes incluidos en el estudio. Durante la hospitalización, 10 (0,87\%) pacientes presentaron complicaciones graves clasificadas como Clavien-Dindo $\geq 3$. En esta serie existió mortalidad en 2 pacientes $(0,17 \%)$, pertenecientes al grupo sometido a GM. Una de ellas presentó un paro cardiorrespiratorio durante el primer día posoperatorio y el otro paciente falleció durante los primeros 30 días después de la cirugía en su casa. En ambos casos consideramos que la mortalidad fue secundaria a un tromboembolismo pulmonar masivo. En la
Tabla 2 se detallan las complicaciones presentadas en ambos tipos de cirugías.

\section{Factores de riesgo para consulta en el servicio de urgencias}

Durante los primeros 30 días después de la cirugía, un 8,03\% $(\mathrm{n}=92)$ de los pacientes tuvo al menos una CU. Los pacientes sometidos a BPG tuvieron una tendencia a consultar más en el SU comparado con los pacientes sometidos a GM, sin embargo, esta diferencia no fue estadísticamente significativa (BPG 9,1\% vs. GM 6,6\%, p = 0,07). Con respecto a la temporalidad de las $\mathrm{CU}$, más de la mitad de ellas ocurrieron durante los primeros 10 días después del alta $(60,8 \%, \mathrm{n}=56)$. Las tres causas más frecuentes de CU fueron dolor abdominal $53 \%(\mathrm{n}=49)$, náuseas y/o vómitos $12 \%(\mathrm{n}=11)$ y problemas relacionados a la herida operatoria $4,3 \%$ $(n=4)$. El análisis univariado identificó que el tiempo operatorio, duración de la hospitalización, IMC preoperatorio y dislipidemia se asociaron a mayor riesgo de CU (Tabla 3). El análisis multivariado identificó que el tiempo operatorio e IMC preopera-

Tabla 2. Complicaciones posoperatorias graves (Clavien-Dindo $\geq 3$ )

\begin{tabular}{lcc|}
\hline Complicaciones Clavien $\geq \mathbf{3}(\mathbf{n}=\mathbf{1 0})$ & $\mathbf{G M}(\mathbf{n}=\mathbf{5 3 3})$ & BPG $(\mathbf{n}=\mathbf{6 1 3})$ \\
Filtración anastomosis & - & $5(0,8 \%)$ \\
Filtración línea de corchetes & $1(0,18 \%)$ & - \\
Hemoperitoneo & $1(0,18 \%)$ & - \\
Hemorragia digestiva alta & - & $1(0,16 \%)$ \\
Mortalidad & $2(0,37 \%)$ & 0 \\
\hline
\end{tabular}


Tabla 3. Análisis univariado de factores asociados a consulta en servicio de urgencias y reingreso hospitalario

\begin{tabular}{|c|c|c|c|c|}
\hline Características & $\begin{array}{c}\text { Consulta en SU } \\
\qquad \mathbf{n}=92\end{array}$ & Valor $\mathbf{p}$ & $\begin{array}{l}\text { Rehospitalización } \\
\qquad \mathrm{n}=42\end{array}$ & Valor $\mathbf{p}$ \\
\hline $\begin{array}{l}\text { Tipo de procedimiento quirúrgico } \\
\text { GM } \\
\text { BPG }\end{array}$ & $\begin{array}{l}36 / 533(6,6 \%) \\
56 / 613(9,1 \%)\end{array}$ & NS & $\begin{array}{l}15 / 533(2,8 \%) \\
27 / 613(4,4 \%)\end{array}$ & NS \\
\hline Tiempo operatorio (mín) & $109 \pm 43$ & 0,001 & $118 \pm 40$ & 0,001 \\
\hline Duración hospitalización (días) & $3,3 \pm 5,8$ & 0,03 & $3,3 \pm 1,7$ & NS \\
\hline Dislipidemia & 8 & 0,039 & 4 & NS \\
\hline Sexo femenino & 72 & NS & 29 & NS \\
\hline DM2 & 17 & NS & 10 & NS \\
\hline Hipertensión & 10 & NS & 4 & NS \\
\hline
\end{tabular}

NS: no significativo.

torio se asociaron de forma independiente a la $\mathrm{CU}$ (Tabla 4).

\section{Factores de riesgo para reingreso hospitalario}

La tasa de reingreso hospitalario durante los primeros 30 días después de la cirugía fue de un 3,7\% $(\mathrm{n}=42)$. Al igual que lo observado con la $\mathrm{CU}$, los pacientes sometidos a BPG tuvieron una tendencia mayor a ser readmitidos comparado con los pacientes sometidos a una GM, no existiendo diferencia estadísticamente significativa entre los grupos (BPG $4,4 \%$ vs. GM 2,8\%, p >0,06). Las tres principales causas de RI en pacientes sometidos a BPG fueron estenosis gastroyeyunal 37\% $(\mathrm{n}=10)$, dolor $\mathrm{ab}-$ dominal $22 \%(n=6)$ y obstrucción intestinal $18 \%$ $(n=5)$. En pacientes sometidos a una GM, las tres principales causas de readmisión fueron dolor abdominal 26,6\% $(n=4)$, trombosis portal $20 \%(n=3)$

Tabla 4. Análisis multivariable para consulta en servicio de urgencias y reingreso hospitalario

\begin{tabular}{|c|c|c|c|c|}
\hline \multirow[t]{2}{*}{ Variable } & \multirow{2}{*}{$\begin{array}{c}\text { Valor } \\
\mathbf{p}\end{array}$} & \multirow{2}{*}{$\begin{array}{l}\text { Odds } \\
\text { Ratio }\end{array}$} & \multicolumn{2}{|c|}{ Intervalo de confianza $95 \%$} \\
\hline & & & Inferior & Superior \\
\hline \multicolumn{5}{|c|}{ Consulta en servicio de urgencias } \\
\hline Tiempo operatorio (mín) & 0,004 & 1,008 & 1,003 & 1,014 \\
\hline IMC preoperatorio $\left(\mathrm{kg} / \mathrm{m}^{2}\right)$ & 0,012 & 1,083 & 1,017 & 1,153 \\
\hline \multicolumn{5}{|l|}{ Reingreso hospitalario } \\
\hline Tiempo operatorio (mín) & 0,005 & 1,011 & 1,003 & 1,019 \\
\hline IMC preoperatorio $\left(\mathrm{kg} / \mathrm{m}^{2}\right)$ & 0,04 & 1,050 & 1,001 & 1,102 \\
\hline
\end{tabular}

y filtración $13 \%(\mathrm{n}=2)$. El análisis univariado identificó que el tiempo operatorio e IMC preoperatorio como factores asociados con RI (Tabla 3). Finalmente, el análisis multivariado identificó como factores independientes asociados al RI el tiempo operatorio e IMC preoperatorio (Tabla 4).

De los 42 pacientes que fueron reingresados, solamente 8 requirieron una intervención quirúrgica, lo que corresponde al $0,7 \%$ de la cohorte total de pacientes y al $19 \%$ de los pacientes reingresados. La Tabla 5 resume los procedimientos realizados. En pacientes sometidos a BPG, 5 necesitaron cirugía lo que corresponde al $0,8 \%$. Un paciente reingresó por apendicitis aguda que fue resuelto sin incidentes por vía laparoscópica. En tres pacientes se realizó aseo quirúrgico laparoscópico e instalación de drenajes por filtración de la línea de corchetes de la bolsa gástrica, con buena evolución posterior. De los 5 pacientes que reingresaron por obstrucción intestinal solo uno requirió cirugía, ya que su obstrucción fue secundaria a una hernia incisional atascada en sitio de inserción del trocar óptico supraumbilical y fue resuelto por laparoscopía. Los otros 4 pacientes presentaron buena evolución con manejo médico. Respecto a los pacientes que reingresaron por estenosis de la anastomosis gastroyeyunal, estos fueron manejados de forma exitosa con dilatación endoscópica con técnica de Savary-Guillard.

En pacientes sometidos a una GM, 3 de ellos requirieron intervención quirúrgica lo que corresponde al $0,6 \%$ de la serie. En una paciente fue necesario convertir la GM a un BPG por estenosis antral, que ocurrió en el inicio de la serie con esta técnica en 
ARTíCULO ORIGINAL

Tabla 5. Procedimientos en pacientes con reingreso hospitalario

\begin{tabular}{|lll|}
\hline Síntomas consulta & Diagnóstico & Cirugía \\
\hline Bypass gástrico & $(\mathbf{n}=\mathbf{5}$ quirúrgicos $)$ & \\
Dolor abdominal & Apendicitis aguda & Apendicectomía laparoscópica \\
Dolor abdominal + distensión abdominal & Hernia incisional complicada & Hernioplastía laparoscópica \\
Dolor abdominal + fiebre & Filtración & Aseo laparoscópico \\
Dolor abdominal & Filtración & Aseo laparoscópico \\
Dolor abdominal + fiebre & Filtración & Aseo laparoscópico \\
Bypass gástrico & $(\mathbf{n}=\mathbf{1 0}$ endoscópicos $)$ & Endoscopía \\
Disfagia, náuseas, vómitos & Estenosis anastomosis gastroyeyunal & Dilatación endoscópica \\
Gastrectomía en manga $(\mathbf{n}=\mathbf{3}$ quirúrgicos) & & \\
Disfagia, náuseas, vómitos & Estenosis antral & Conversión a BPG \\
Dolor abdominal + fiebre & Filtración línea de grapado & Aseo laparoscópico + prótesis esofágica \\
Dolor abdominal + fiebre & Filtración línea de grapado & Aseo laparoscópico + prótesis esofágica \\
\hline
\end{tabular}

el periodo 2006-2007. En otros dos pacientes fue necesario realizar un aseo quirúrgico laparoscópico e instalación de prótesis por filtración de en la línea de corchetes.

\section{Discusión}

En este estudio analizamos las CU y los RI en pacientes con obesidad sometidos a $\mathrm{CB}$ primaria durante los primeros 30 días después del alta. Adicionalmente, buscamos identificar variables que pudieran estar asociadas a estos indicadores. La CU después de una $\mathrm{CB}$ es un indicador importante de calidad. En nuestro estudio, un 8,03\% de los pacientes presentó una CU durante los primeros 30 días después de la cirugía, coincidiendo con lo reportado en la literatura que varía entre un 7,5\%-10,7\%6,18,19. Las variables independientes asociadas a la CU fueron el tiempo operatorio e IMC preoperatorio. En este trabajo el tipo de cirugía no fue un factor asociado a la $\mathrm{CU}$, coincidiendo con lo publicado por Iskra et al. ${ }^{20}$. Sin embargo, contrasta con lo reportado en otros estudios en los cuales pacientes sometidos a BPG tienen mayores $\mathrm{CU}$ que los pacientes sometidos una $\mathrm{GM}^{18,19}$. En nuestra serie, los pacientes sometidos a BPG tuvieron una tendencia a tener mayor cantidad de CU que los sometidos a GM, sin embargo, esta diferencia no fue estadísticamente significativa, pudiendo ser explicada por el número reducido de pacientes incluidos en el análisis debido al bajo porcentaje de consultas.

Las causas más frecuentes de CU fueron dolor abdominal, nauseas/vómitos y problemas asociados con la herida operatoria. Estos motivos de consulta concuerdan con lo reportado en diferentes estudios demostrando lo frecuente de estos síntomas durante el primer mes después de una $\mathrm{CB}^{18,19,21,22}$. De igual forma, una proporción significativa de los pacientes que consultaron en la urgencia no necesitaron ser hospitalizados, lo que indica que la mayoría de las causas de consulta pueden tratarse exitosamente sin necesidad de requerir hospitalización. Si finalmente muchas de estas visitas pudieron haber sido prevenidas $\mathrm{y}$, por lo tanto, haber sido innecesarias como lo han reportado otros estudios ${ }^{4,23}$, no fue analizado en este trabajo.

El RI es también un indicador de calidad importante, no solo por el efecto que este genera en el paciente y a su equipo tratante, sino que también por los costos asociados que implica una hospitalización no programada ${ }^{14}$. Variables como el tipo de cirugía realizada, el abordaje usado, duración de la cirugía, complicaciones posoperatorias, entre otras han sido asociadas a reingreso $0^{5,24,25}$. En relación al tipo cirugía realizada los resultados son controversiales. Algunos trabajos muestran mayores tasas de reingreso después de un BPG que luego de una GM con tasas promedio de un $6 \%$ y $4 \%$, respectivamente ${ }^{5,12,24,26}$, mientras que, en este trabajo, al igual que el publicado por Doumouras et al..$^{22}$ no se encontraron diferencias estadísticamente significativas entre ambas cirugías, pese a existir una tendencia a mayor reingreso después del BPG. De las variables perioperatorias estudiadas solo el tiempo operatorio se asoció de forma independiente al reingreso, coincidiendo 
con lo reportado por Tayne et al. ${ }^{25}$. Existen otras características de los pacientes al momento de la cirugía como la edad, IMC preoperatorio, ASA IIIIV, uso de esteroides, hipertensión arterial y uso de insulina que pueden asociarse al reingreso ${ }^{5,7,24,27,28}$. En nuestro estudio encontramos que el grado de obesidad, determinado por el IMC preoperatorio, se asoció de forma independiente al reingreso. Una posible explicación podría ser que en pacientes con mayores grados de obesidad es habitual tener tiempos operatorios más prolongados que puede deberse a una mayor cantidad de tejido adiposo visceral y/o existencia de un lóbulo hepático izquierdo más prominente dificultando y enlenteciendo la cirugía. Aunque la evidencia es controversial ${ }^{29}$, en nuestra práctica fomentamos la pérdida de peso preoperatoria, ya que puede facilitar la cirugía disminuyendo el volumen hepático y grasa visceral.

Nuestro estudio tiene fortalezas y limitaciones. Una de las fortalezas es que a nuestro saber representa la mayor experiencia nacional destinada a caracterizar las CU y RI después de una CB primaria. El carácter retrospectivo de este estudio representa una limitación. Con la idea de reducir la falta potencial de datos, decidimos enfocarnos en la CU y RI durante los primeros 30 días después de la cirugía, ya que en nuestra experiencia durante el primer mes los pacientes tienden a consultar en el centro donde se realizaron la cirugía. Sin embargo, no es posible descartar que puedan existir eventos no considerados en este estudio en pacientes que hayan sido atendidos en otros centros subestimando la frecuencia de estos indicadores. A pesar de estas limitaciones, los resultados presentados en este trabajo concuerdan con los resultados reportados por otros estudios.

\section{Conclusiones}

Después de una cirugía bariátrica primaria una baja proporción de pacientes consulta en urgencia y requiere ser hospitalizado lo que demuestra la seguridad de este tipo de intervenciones. En nuestro estudio, tanto pacientes con mayores grados de obesidad preoperatorios, así como pacientes con tiempos operatorios más prolongados tendrían un mayor riesgo de tener consultas de urgencia y/o un reingreso. Parece razonable, en este grupo de pacientes, enfatizar la educación con respecto a los síntomas más comunes en el posoperatorio y aquellos que ameritan una consulta en la urgencia, así como tener canales óptimos de comunicación con equipo tratante a fin reducir consultas que podrían ser consideradas no necesarias.

\section{Responsabilidades éticas}

Protección de personas y animales. Los autores declaran que para esta investigación no se han realizado experimentos en seres humanos ni en animales.

Confidencialidad de los datos. Los autores declaran que en este artículo no aparecen datos de pacientes.

Conflictos de interés: no hay.

Financiamiento: No hay fuentes de financiamiento involucradas.

\section{Bibliografía}

1. Carlsson LMS, Sjoholm K, Jacobson $\mathrm{P}$, Andersson-Assarsson JC, Svensson PA, Taube M, et al. Life Expectancy after Bariatric Surgery in the Swedish Obese Subjects Study. N Engl J Med. 2020;383:1535-43.

2. Aminian A, Jamal MH, Andalib A, Batayyah E, Romero-Talamas H, Chand B, et al. Is Laparoscopic Bariatric Surgery a Safe Option in Extremely High-Risk Morbidly Obese Patients? J Laparoendosc Adv Surg Tech A. 2015;25:707-11.

3. Aminian A, Brethauer SA, Kirwan JP, Kashyap SR, Burguera B, Schauer PR.
How safe is metabolic/diabetes surgery? Diabetes Obes Metab. 2015;17:198-201.

4. Chen J, Mackenzie J, Zhai Y, O'Loughlin J, Kholer R, Morrow E, et al. Preventing Returns to the Emergency Department Following Bariatric Surgery. Obes Surg. 2017;27:1986-92.

5. Sippey M, Kasten KR, Chapman WH, Pories WJ, Spaniolas K. 30-day readmissions after sleeve gastrectomy versus Roux-en-Y gastric bypass. Surg Obes Relat Dis. 2016;12:991-6.

6. Telem DA, Yang J, Altieri M, Patterson W, Peoples B, Chen H, et al. Rates and Risk Factors for Unplanned Emergency Department Utilization and Hospital
Readmission Following Bariatric Surgery. Ann Surg. 2016;263:956-60.

7. Sethi M, Patel K, Zagzag J, Parikh M, Saunders J, Ude-Welcome A, et al. ThirtyDay Readmission After Laparoscopic Sleeve Gastrectomy-a Predictable Event? J Gastrointest Surg. 2016;20:244-52.

8. Encinosa WE, Bernard DM, Chen CC, Steiner CA. Healthcare utilization and outcomes after bariatric surgery. Med Care 2006;44:706-12.

9. Dorman RB, Miller CJ, Leslie DB, Serrot FJ, Slusarek B, Buchwald H, et al. Risk for hospital readmission following bariatric surgery. PLoS One. 2012;7:e32506. 
10. Hong B, Stanley E, Reinhardt S, Panther K, Garren MJ, Gould JC. Factors associated with readmission after laparoscopic gastric bypass surgery. Surg Obes Relat Dis. 2012;8:691-5.

11. Saunders JK, Ballantyne GH, Belsley S, Stephens D, Trivedi A, Ewing DR, et al. 30-day readmission rates at a high volume bariatric surgery center: laparoscopic adjustable gastric banding, laparoscopic gastric bypass, and vertical banded gastroplasty-Roux-en-Y gastric bypass. Obes Surg. 2007;17:1171-7.

12. Patterson WL, Peoples BD, Gesten FC. Predicting potentially preventable hospital readmissions following bariatric surgery. Surg Obes Relat Dis. 2015;11:866-72.

13. Lawson EH, Hall BL, Louie R, Ettner SL, Zingmond DS, Han L, et al. Association between occurrence of a postoperative complication and readmission: implications for quality improvement and cost savings. Ann Surg. 2013;258:10-8.

14. Postel M, Frank PN, Barry T, Satou N, Shemin R, Benharash P. The cost of preventing readmissions: why surgeons should lead the effort. Am Surg. 2014;80:1003-6.

15. Quezada N, Leon F, Jones A, Varas J, Funke R, Crovari F, et al. High frequency of internal hernias after Roux-en-Y gastric bypass. Obes Surg. 2015;25:615-21.

16. Boza C, Daroch D, Barros D, Leon F, Funke R, Crovari F. Long-term outcomes of laparoscopic sleeve gastrectomy as a primary bariatric procedure. Surg Obes Relat Dis. 2014;10:1129-33.

17. American Society for M, Bariatric
Surgery Clinical Issues C. ASMBS updated position statement on prophylactic measures to reduce the risk of venous thromboembolism in bariatric surgery patients. Surg Obes Relat Dis. 2013;9:493-7.

18. Macht R, George J, Ameli O, Hess D, Cabral H, Kazis L. Factors associated with bariatric postoperative emergency department visits. Surg Obes Relat Dis. 2016;12:1826-31.

19. Mora-Pinzon MC, Henkel D, Miller RE, Remington PL, Gould JC, Kothari SN, et al. Emergency department visits and readmissions within 1 year of bariatric surgery: A statewide analysis using hospital discharge records. Surgery. 2017;162:1155-62.

20. Iskra MP, Ramon JM, Martinez-Serrano A, Serra C, Goday A, Trillo L, et al. Unplanned emergency department consultations and readmissions within 30 and 90 days of bariatric surgery. Cir Esp. 2018;96:221-5.

21. Ahmed A, AlBuraikan D, B AL, AlJohi W, Alanazi W, AlRasheed B. Readmissions and Emergency Department Visits after Bariatric Surgery at Saudi Arabian Hospital: The Rates, Reasons, and Risk Factors. Obesity facts. 2017;10:432-43.

22. Doumouras AG, Saleh F, Hong D. 30Day readmission after bariatric surgery in a publicly funded regionalized center of excellence system. Surg Endosc. 2016;30:2066-72.

23. Kellogg TA, Swan T, Leslie DA, Buchwald H, Ikramuddin S. Patterns of readmission and reoperation within 90 days after Roux-en-Y gastric bypass. Surg Obes Relat Dis. 2009;5:416-23.

24. Khorgami Z, Andalib A, Aminian A, Kroh MD, Schauer PR, Brethauer SA. Predictors of readmission after laparoscopic gastric bypass and sleeve gastrectomy: a comparative analysis of ACS-NSQIP database. Surg Endosc. 2016;30:2342-50.

25. Tayne S, Merrill CA, Shah SN, Kim J, Mackey WC. Risk factors for 30-day readmissions and modifying postoperative care after gastric bypass surgery. J Am Coll Surg. 2014;219:489-95.

26. Berger ER, Huffman KM, Fraker T, Petrick AT, Brethauer SA, Hall BL, et al. Prevalence and Risk Factors for Bariatric Surgery Readmissions: Findings From 130,007 Admissions in the Metabolic and Bariatric Surgery Accreditation and Quality Improvement Program. Ann Surg. 2018;267:122-31. doi: 10.1097/ SLA.0000000000002079.

27. Willson TD, Gomberawalla A, Mahoney K, Lutfi RE. Factors influencing 30day emergency visits and readmissions after sleeve gastrectomy: results from a community bariatric center. Obes Surg. 2015;25:975-81.

28. Aminian A, Brethauer SA, Sharafkhah M, Schauer PR. Development of a sleeve gastrectomy risk calculator. Surg Obes Relat Dis. 2015;11:758-64.

29. Cassie S, Menezes C, Birch DW, Shi X, Karmali S. Effect of preoperative weight loss in bariatric surgical patients: a systematic review. Surg Obes Relat Dis. 2011;7:760-7; discussion 7. 